\title{
Expanding the definition of multivariate correlation
}

\author{
W. Conley \\ Departments of Mathematics and Business Administration, \\ University of Wisconsin at Green Bay, USA
}

\begin{abstract}
The complexities of large scale data analysis, in our computer age, invite the development of new sophisticated statistics to help in this task. One entry into this arena is the CTSP multivariate correlation statistic. A five variable 49 line spreadsheet of data was analyzed using CTSP by Conley and the relationship was found to be linear. Presented here is a much larger example involving nine variables and 89 lines of data, where CTSP reveals a correlation that is nonlinear (exponential in this case). Specifically, nine columns (representing nine variables) of 89 lines of data are being analyzed to see if the variables they represent are correlated in some fashion (linear or nonlinear). Therefore, the data is read into the CTSP statistical analysis simulation program which is adjusted for nine variables and 89 lines of data. Then, using a generalization of the Pythagorean distance measure to nine dimensions, a shortest route connecting the 89 points in a closed loop tour is calculated. Then several random data sets of 9 × 89 size (in similar ranges to the original data) are generated and the shortest routes are calculated for them. In this case, the actual data had a much shorter shortest route than the random data's shortest routes. Therefore, statistically speaking it can be argued that the actual data is correlated in some fashion because it is following a pattern and hence the points are more compact (closer together in nine dimensional space) leading to a shorter shortest route. The relationship is exponential in this case. This expanded view of correlation (linear or nonlinear) can complement the standard linear analysis Anderson currently uses. Additionally, a second example involving eight variables is presented for comparison purposes.
\end{abstract}

Keywords: multivariate correlation, CTSP statistic, shortest route statistical test, linear and nonlinear analysis. 


\section{Introduction}

The new CTSP correlation statistic was developed in 2002 to help discover multivariate relationships whether they be linear or nonlinear. The CTSP is short for (an acronym) correlation using the travelling salesman problem. The idea in two dimensions can be illustrated by thinking of $X, Y$ pairs of points that are following the shape of a parabola when graphed. These points will have a shorter shortest route connecting them, than the shortest route connecting the same number of random points (in the same range as the parabola points).

A three dimensional example would be $X, Y, Z$ triples on a flat plane going through the points $(0,0,0),(100,100,100),(100,0,100)$, and $(0,100,0)$ in the region 0 to 100 for all three variables. The shortest route connecting these $X, Y$, $Z$ triples (say 75 of them, for example) would be much shorter than the shortest route connecting 75 random triples (in the same range) that are not following a pattern.

This can be exploited statistically to show a correlation (or lack thereof) when analyzing multivariate data. The name CTSP statistic comes from the mathematically famous TSP problems (so called travelling salesman problem) of finding a shortest route to connect $n$ points in a closed loop tour.

This analysis presented here uses the multi stage Monte Carlo optimization MSMCO TSP algorithm for the statistical analysis. Let us look at an example.

\section{Numeric example one}

Researches believe that the nine variables represented by the nine columns of data in Table 1 are correlated in a linear or nonlinear fashion.

They think that the first eight variables are perhaps driving the ninth variable ( $X_{9}$ whose data is in Column 9). Therefore, to test the null hypothesis of no correlation between the nine variables $\left(X_{1}, X_{2} \ldots X_{9}\right)$ versus the alternative hypothesis of correlation, the 89 lines of 9 columns of data are read into the MSMSCO TSP algorithm program adjusted for 89 lines of data and nine variables. The Pythagorean theorem distance measure is used after expanding it to nine dimensional distance calculations.

A few seconds to a minute of computer run time (on a desktop PC) yielded a shortest route of total distance 5,776.308 (see Table 2 for the route read left to right) connecting the 89 nine dimensional points in a closed loop tour.

Then four sets of 89 lines of nine columns of random data (in similar 0-100 ranges as the actual data) were read into the MSMCO TSP algorithm. Their shortest routes were calculated to be $6786.314,6518.579,6600.060$ and 6410.455. Therefore,

$$
C T S P=A / B=5776.308 /(6518.579+6600.060) / 2=.88
$$

where $\mathrm{A}$ is the shortest route distance for the actual data and $\mathrm{B}$ is the median of the four random data shortest routes. Now taking the $3 \times 4=12 \mathrm{~A}_{i} / \mathrm{B}_{i}$ quotients using all combinations of the four random shortest routes, we see that their range 
Table 1: $\quad$ The data.

\begin{tabular}{|c|c|c|c|c|c|c|c|c|c|}
\hline Point & $X_{1}$ & $X_{2}$ & $X_{3}$ & $X_{4}$ & $X_{5}$ & $X_{6}$ & $X_{7}$ & $X_{8}$ & $X_{9}$ \\
\hline 1 & 73 & 12 & 46 & 59 & 41 & 24 & 95 & 96 & 3.6 \\
\hline 2 & 66 & 5 & 69 & 49 & 94 & 18 & 100 & 21 & 4.9 \\
\hline 3 & 9 & 17 & 18 & 58 & 85 & 85 & 62 & 29 & 3 \\
\hline 4 & 40 & 41 & 95 & 91 & 8 & 68 & 18 & 23 & 3.6 \\
\hline 5 & 40 & 70 & 58 & 36 & 8 & 35 & 30 & 72 & 2.7 \\
\hline 6 & 5 & 51 & 49 & 44 & 37 & 33 & 72 & 23 & 2 \\
\hline 7 & 0 & 50 & 89 & 77 & 83 & 13 & 64 & 52 & 5.1 \\
\hline 8 & 63 & 99 & 29 & 13 & 27 & 46 & 30 & 82 & 3.7 \\
\hline 9 & 61 & 56 & 100 & 14 & 91 & 1 & 25 & 31 & 3.4 \\
\hline 10 & 28 & 70 & 57 & 30 & 86 & 31 & 47 & 100 & 6 \\
\hline 11 & 31 & 100 & 11 & 99 & 73 & 76 & 5 & 82 & 7.5 \\
\hline 12 & 50 & 60 & 16 & 79 & 11 & 86 & 100 & 43 & 5.8 \\
\hline 13 & 65 & 23 & 77 & 39 & 65 & 36 & 51 & 87 & 5.7 \\
\hline 14 & 32 & 13 & 100 & 90 & 18 & 33 & 38 & 29 & 2.8 \\
\hline 15 & 100 & 10 & 95 & 60 & 24 & 24 & 33 & 88 & 5.3 \\
\hline 16 & 40 & 94 & 94 & 30 & 70 & 46 & 65 & 82 & 10.7 \\
\hline 17 & 52 & 20 & 67 & 14 & 3 & 99 & 41 & 94 & 3.8 \\
\hline 18 & 68 & 89 & 29 & 2 & 62 & 38 & 92 & 100 & 7.7 \\
\hline 19 & 17 & 90 & 72 & 40 & 16 & 49 & 40 & 4 & 2.3 \\
\hline 20 & 71 & 48 & 66 & 71 & 36 & 1 & 99 & 53 & 5.8 \\
\hline 21 & 78 & 56 & 57 & 44 & 62 & 82 & 39 & 19 & 5.5 \\
\hline 22 & 70 & 47 & 75 & 25 & 100 & 70 & 20 & 20 & 5.1 \\
\hline 23 & 46 & 0 & 37 & 70 & 88 & 100 & 34 & 20 & 5.4 \\
\hline 24 & 58 & 39 & 22 & 97 & 68 & 57 & 42 & 52 & 5.4 \\
\hline 25 & 65 & 61 & 93 & 5 & 31 & 33 & 29 & 81 & 4 \\
\hline 26 & 88 & 90 & 62 & 90 & 86 & 17 & 69 & 42 & 12.9 \\
\hline 27 & 51 & 28 & 20 & 77 & 75 & 64 & 29 & 13 & 2.9 \\
\hline 28 & 57 & 74 & 36 & 71 & 100 & 74 & 57 & 91 & 14.6 \\
\hline 29 & 12 & 77 & 41 & 72 & 63 & 48 & 36 & 92 & 5.7 \\
\hline 30 & 33 & 3 & 32 & 63 & 88 & 57 & 81 & 73 & 5.2 \\
\hline 31 & 100 & 97 & 98 & 32 & 45 & 85 & 0 & 70 & 11.2 \\
\hline 32 & 4 & 19 & 69 & 33 & 100 & 9 & 63 & 88 & 3.6 \\
\hline 33 & 13 & 23 & 25 & 42 & 27 & 96 & 21 & 92 & 2.5 \\
\hline 34 & 38 & 26 & 21 & 100 & 1 & 26 & 40 & 5 & 1.3 \\
\hline 35 & 77 & 57 & 72 & 19 & 97 & 91 & 91 & 44 & 13.3 \\
\hline 36 & 52 & 63 & 10 & 18 & 22 & 68 & 100 & 17 & 2.7 \\
\hline 37 & 55 & 19 & 35 & 41 & 32 & 37 & 35 & 82 & 2.4 \\
\hline 38 & 11 & 41 & 42 & 89 & 46 & 5 & 35 & 57 & 2.3 \\
\hline 39 & 36 & 20 & 10 & 87 & 4 & 86 & 58 & 95 & 3.9 \\
\hline 40 & 65 & 66 & 15 & 23 & 0 & 3 & 16 & 67 & 1.3 \\
\hline 41 & 50 & 58 & 3 & 23 & 100 & 97 & 73 & 92 & 8.8 \\
\hline 42 & 14 & 79 & 29 & 88 & 56 & 72 & 34 & 75 & 5.9 \\
\hline 43 & 84 & 44 & 35 & 21 & 24 & 54 & 2 & 57 & 2.2 \\
\hline
\end{tabular}


Table 1: $\quad$ Continued.

\begin{tabular}{|c|c|c|c|c|c|c|c|c|c|}
\hline Point & $X_{1}$ & $X_{2}$ & $X_{3}$ & $X_{4}$ & $X_{5}$ & $X_{6}$ & $X_{7}$ & $X_{8}$ & $X_{9}$ \\
\hline 44 & 28 & 32 & 44 & 80 & 88 & 13 & 21 & 10 & 2.1 \\
\hline 45 & 51 & 40 & 100 & 22 & 79 & 61 & 74 & 99 & 11.2 \\
\hline 46 & 51 & 94 & 2 & 4 & 6 & 100 & 27 & 88 & 3.3 \\
\hline 47 & 56 & 2 & 31 & 60 & 74 & 12 & 37 & 1 & 1.5 \\
\hline 48 & 35 & 88 & 5 & 79 & 1 & 36 & 98 & 48 & 3.8 \\
\hline 49 & 83 & 95 & 27 & 46 & 32 & 90 & 40 & 59 & 7.2 \\
\hline 50 & 40 & 4 & 85 & 50 & 56 & 99 & 85 & 42 & 6.6 \\
\hline 51 & 90 & 43 & 73 & 52 & 76 & 92 & 74 & 36 & 12.1 \\
\hline 52 & 33 & 23 & 53 & 2 & 61 & 34 & 74 & 30 & 2 \\
\hline 53 & 55 & 100 & 9 & 79 & 70 & 35 & 0 & 3 & 2.7 \\
\hline 54 & 45 & 51 & 3 & 83 & 48 & 63 & 49 & 37 & 3.4 \\
\hline 55 & 43 & 100 & 31 & 87 & 31 & 34 & 25 & 55 & 4.3 \\
\hline 56 & 96 & 7 & 13 & 16 & 81 & 27 & 66 & 44 & 2.7 \\
\hline 57 & 13 & 43 & 37 & 41 & 19 & 59 & 77 & 35 & 2.2 \\
\hline 58 & 60 & 58 & 14 & 90 & 93 & 65 & 75 & 74 & 11.4 \\
\hline 59 & 18 & 28 & 6 & 97 & 1 & 76 & 22 & 81 & 2.3 \\
\hline 60 & 29 & 29 & 15 & 32 & 60 & 65 & 64 & 6 & 1.8 \\
\hline 61 & 57 & 63 & 75 & 91 & 81 & 83 & 86 & 80 & 22.9 \\
\hline 62 & 43 & 57 & 60 & 53 & 90 & 62 & 90 & 86 & 12.6 \\
\hline 63 & 57 & 95 & 50 & 82 & 35 & 100 & 90 & 33 & 12.7 \\
\hline 64 & 29 & 100 & 45 & 66 & 92 & 63 & 78 & 100 & 16.3 \\
\hline 65 & 81 & 100 & 1 & 18 & 99 & 6 & 90 & 62 & 6.4 \\
\hline 66 & 41 & 31 & 33 & 16 & 13 & 35 & 90 & 8 & 1.4 \\
\hline 67 & 93 & 47 & 79 & 48 & 100 & 76 & 60 & 91 & 19.2 \\
\hline 68 & 45 & 27 & 70 & 65 & 79 & 92 & 56 & 26 & 6.6 \\
\hline 69 & 7 & 95 & 77 & 30 & 12 & 10 & 56 & 36 & 2.2 \\
\hline 70 & 31 & 72 & 51 & 73 & 88 & 75 & 82 & 25 & 8.8 \\
\hline 71 & 11 & 20 & 19 & 43 & 82 & 100 & 95 & 58 & 5.1 \\
\hline 72 & 95 & 7 & 31 & 15 & 26 & 12 & 51 & 89 & 2.3 \\
\hline 73 & 62 & 23 & 3 & 17 & 71 & 60 & 42 & 20 & 1.8 \\
\hline 74 & 66 & 74 & 60 & 10 & 41 & 52 & 46 & 17 & 3.1 \\
\hline 75 & 88 & 53 & 46 & 9 & 65 & 94 & 82 & 68 & 9.4 \\
\hline 76 & 8 & 10 & 92 & 39 & 37 & 83 & 55 & 3 & 2.3 \\
\hline 77 & 66 & 43 & 81 & 93 & 39 & 27 & 21 & 95 & 6.8 \\
\hline 78 & 31 & 60 & 79 & 37 & 33 & 75 & 46 & 25 & 3.6 \\
\hline 79 & 62 & 40 & 100 & 48 & 91 & 6 & 55 & 66 & 7 \\
\hline 80 & 29 & 83 & 24 & 28 & 56 & 80 & 77 & 63 & 5.6 \\
\hline 81 & 35 & 36 & 9 & 39 & 49 & 43 & 28 & 96 & 2.4 \\
\hline 82 & 17 & 40 & 81 & 23 & 36 & 11 & 55 & 14 & 1.5 \\
\hline 83 & 44 & 56 & 46 & 84 & 91 & 100 & 19 & 10 & 6.1 \\
\hline 84 & 1 & 20 & 100 & 20 & 34 & 16 & 2 & 74 & 1.4 \\
\hline 85 & 2 & 56 & 0 & 20 & 15 & 12 & 35 & 100 & 1.1 \\
\hline 86 & 98 & 77 & 100 & 19 & 48 & 8 & 35 & 35 & 4.8 \\
\hline
\end{tabular}


Table 1: $\quad$ Continued.

\begin{tabular}{cccccccccc}
\hline Point & $X_{1}$ & $X_{2}$ & $X_{3}$ & $X_{4}$ & $X_{5}$ & $X_{6}$ & $X_{7}$ & $X_{8}$ & $X_{9}$ \\
\hline 87 & 72 & 56 & 4 & 39 & 97 & 15 & 60 & 8 & 2.8 \\
88 & 39 & 4 & 94 & 98 & 60 & 2 & 100 & 74 & 7.2 \\
89 & 97 & 16 & 59 & 100 & 81 & 95 & 52 & 89 & 18.5 \\
\hline
\end{tabular}

Table 2: $\quad$ The route.

\begin{tabular}{cccccccccc}
\hline 43 & 17 & 33 & 39 & 59 & 54 & 24 & 58 & 28 & 64 \\
29 & 42 & 11 & 55 & 53 & 27 & 83 & 23 & 68 & 50 \\
76 & 78 & 19 & 69 & 82 & 6 & 57 & 66 & 36 & 48 \\
12 & 63 & 70 & 26 & 79 & 9 & 86 & 74 & 52 & 2 \\
1 & 20 & 88 & 7 & 32 & 10 & 16 & 18 & 65 & 87 \\
47 & 44 & 38 & 34 & 4 & 14 & 77 & 15 & 13 & 45 \\
67 & 89 & 61 & 62 & 30 & 71 & 3 & 60 & 73 & 56 \\
72 & 37 & 81 & 85 & 40 & 5 & 8 & 46 & 49 & 80 \\
41 & 75 & 35 & 51 & 21 & 22 & 31 & 25 & 85 & 43 \\
\hline
\end{tabular}

is $6410.455 / 6786.314=.945$ to $6786.314 / 6410.455=1.059$. This range contains the vast majority of the sampling distribution of CTSP under the null hypothesis of no correlation. However, from eqn (1) our CTSP $=.88$, which is less than this range. Therefore, the null hypothesis of no correlation can be confidently rejected. The nine variables are correlated and the equation

$$
X_{9}=.1666 \exp \left(\left(X_{1}+X_{2}+X_{3}+X_{4}+X_{5}+X_{6}+X_{7}+X_{8}\right) / 125\right)
$$

fits the data fairly well.

It should be noted that the range of the actual $X_{9}$ variable values is somewhat smaller (0-25) rather than 0-100 for the other eight variables. However, if one evaluates eqn (2) with 100 for $X_{1}$ through $X_{8}, X_{9}$ is 100 . Therefore, the restricted range of $X_{9}$ was a function of the correlation, so random sampling all nine ranges in the area 0-100 seems appropriate for the randomness comparison.

However, if the researcher or engineer believes that there is scientific justification for having different ranges for the variables, they can be used for the random data sets generation and subsequent testing. The engineer or scientist will know what ranges are believable and appropriate for the application at hand.

The central point is that a shorter shortest route (than randomly generated data's shortest routes) will indicate that the data is following a pattern and is hence indicating that the variables are correlated.

\section{Example two}

Researchers studying the eight columns of $n=49$ lines of data in Table 3 want to test the hypothesis of no correlation between the eight variables represented by the eight columns of data. 
Therefore, the $8 \times 49$ array is read into the MSMCO TSP algorithm. A less than one minute computer run produces a shortest route of total distance $\mathrm{A}=$ 3571.170 (see Table 4) for analysis.

Table 3: Example two data.

\begin{tabular}{|c|c|c|c|c|c|c|c|c|}
\hline Point & $X_{1}$ & $X_{2}$ & $X_{3}$ & $X_{4}$ & $X_{5}$ & $X_{6}$ & $X_{7}$ & $X_{8}$ \\
\hline 1 & 55 & 10 & 89 & 17 & 33 & 50 & 100 & 45 \\
\hline 2 & 27 & 38 & 86 & 67 & 50 & 12 & 43 & 47 \\
\hline 3 & 82 & 98 & 61 & 13 & 51 & 81 & 27 & 61 \\
\hline 4 & 72 & 74 & 4 & 93 & 100 & 83 & 12 & 56 \\
\hline 5 & 37 & 30 & 57 & 70 & 15 & 90 & 86 & 17 \\
\hline 6 & 4 & 100 & 50 & 6 & 3 & 66 & 47 & 4 \\
\hline 7 & 94 & 50 & 82 & 39 & 19 & 79 & 84 & 3 \\
\hline 8 & 92 & 99 & 83 & 23 & 21 & 27 & 77 & 4 \\
\hline 9 & 46 & 100 & 82 & 1 & 4 & 12 & 38 & 10 \\
\hline 10 & 0 & 18 & 7 & 85 & 24 & 12 & 64 & 62 \\
\hline 11 & 85 & 61 & 21 & 100 & 100 & 17 & 23 & 89 \\
\hline 12 & 74 & 70 & 70 & 100 & 100 & 94 & 57 & 18 \\
\hline 13 & 38 & 84 & 59 & 17 & 98 & 9 & 11 & 87 \\
\hline 14 & 57 & 22 & 36 & 19 & 2 & 47 & 49 & 82 \\
\hline 15 & 54 & 26 & 92 & 3 & 26 & 25 & 19 & 99 \\
\hline 16 & 40 & 52 & 60 & 97 & 38 & 96 & 20 & 93 \\
\hline 17 & 71 & 56 & 10 & 82 & 79 & 52 & 22 & 87 \\
\hline 18 & 88 & 57 & 75 & 42 & 2 & 47 & 56 & 15 \\
\hline 19 & 100 & 37 & 45 & 42 & 55 & 52 & 26 & 3 \\
\hline 20 & 47 & 8 & 41 & 67 & 36 & 29 & 59 & 87 \\
\hline 21 & 16 & 45 & 20 & 25 & 71 & 99 & 53 & 62 \\
\hline 22 & 36 & 63 & 46 & 28 & 67 & 40 & 61 & 3 \\
\hline 23 & 21 & 100 & 13 & 94 & 22 & 20 & 21 & 52 \\
\hline 24 & 29 & 51 & 41 & 100 & 39 & 36 & 75 & 21 \\
\hline 25 & 59 & 85 & 83 & 41 & 27 & 1 & 79 & 40 \\
\hline 26 & 54 & 72 & 49 & 62 & 32 & 58 & 55 & 15 \\
\hline 27 & 65 & 40 & 71 & 65 & 69 & 34 & 3 & 27 \\
\hline 28 & 10 & 45 & 73 & 30 & 49 & 21 & 98 & 84 \\
\hline 29 & 23 & 65 & 76 & 80 & 5 & 44 & 6 & 57 \\
\hline 30 & 48 & 15 & 80 & 28 & 80 & 11 & 84 & 89 \\
\hline 31 & 55 & 42 & 70 & 40 & 28 & 17 & 56 & 76 \\
\hline 32 & 66 & 7 & 82 & 100 & 98 & 73 & 80 & 33 \\
\hline 33 & 20 & 8 & 71 & 37 & 7 & 20 & 55 & 39 \\
\hline 34 & 74 & 63 & 26 & 9 & 33 & 4 & 44 & 14 \\
\hline 35 & 80 & 64 & 81 & 15 & 99 & 38 & 72 & 86 \\
\hline 36 & 26 & 58 & 5 & 35 & 56 & 17 & 21 & 75 \\
\hline 37 & 24 & 40 & 26 & 2 & 90 & 100 & 3 & 53 \\
\hline 38 & 9 & 54 & 31 & 18 & 33 & 41 & 49 & 26 \\
\hline 39 & 64 & 88 & 54 & 56 & 61 & 68 & 69 & 8 \\
\hline
\end{tabular}


Table 3: $\quad$ Continued.

\begin{tabular}{ccccccccc}
\hline Point & $X_{1}$ & $X_{2}$ & $X_{3}$ & $X_{4}$ & $X_{5}$ & $X_{6}$ & $X_{7}$ & $X_{8}$ \\
\hline 40 & 78 & 21 & 56 & 37 & 35 & 86 & 92 & 92 \\
41 & 32 & 53 & 34 & 39 & 28 & 29 & 25 & 99 \\
42 & 61 & 88 & 82 & 10 & 61 & 61 & 23 & 7 \\
43 & 89 & 44 & 92 & 48 & 91 & 6 & 58 & 83 \\
44 & 61 & 48 & 54 & 100 & 8 & 88 & 34 & 44 \\
45 & 98 & 100 & 55 & 29 & 97 & 1 & 83 & 62 \\
46 & 32 & 96 & 70 & 87 & 95 & 0 & 89 & 8 \\
47 & 100 & 93 & 47 & 73 & 17 & 75 & 81 & 17 \\
48 & 85 & 47 & 39 & 25 & 5 & 14 & 33 & 71 \\
49 & 13 & 88 & 96 & 98 & 39 & 88 & 68 & 37 \\
\hline
\end{tabular}

Table 4: $\quad$ Example two route.

\begin{tabular}{cccccccccc}
\hline 45 & 35 & 43 & 30 & 28 & 31 & 20 & 10 & 24 & 49 \\
5 & 44 & 16 & 29 & 23 & 11 & 17 & 4 & 12 & 32 \\
46 & 25 & 8 & 18 & 7 & 47 & 26 & 39 & 22 & 38 \\
6 & 9 & 42 & 3 & 37 & 21 & 40 & 1 & 33 & 2 \\
27 & 19 & 34 & 48 & 14 & 15 & 13 & 41 & 36 & \\
\hline
\end{tabular}

Then four sets of random data of $8 \times 49$ are read into the MSMCO TSP algorithm. Their shortest routes turn out to be $3346.265,3473.206,3564.802$ and 3363.000. Note that the real data shortest route distance (3571.170) is comparable to these and will not produce a relatively smaller CTSP. Therefore, the null hypothesis of no correlation between the variables cannot be rejected.

\section{Expanded definition of correlation}

We are now prepared with the CTSP multivariate statistic (for the computer age) to expand our definition of multivariate correlation to the discovery of any type of pattern, whether it be linear, nonlinear and even non-functional (like a mathematical relation). Additionally, if one wishes to assume and/or test for various underlying distributions, CTSP can help in that accommodation also.

\section{Conclusion}

Much of the standard multivariate analysis used today assumes sampling from multivariate normal distributions and looking for linear relationships. However, with CTSP we can go beyond this and look for any type of relationship that is revealed by a relatively shorter shortest route through the $k$ dimensional space (for $k$ variables) when compared with similar random data. 
The various TSP algorithms can help trucking companies and transportation entities in their deliveries to their valued customers. However, the TSP analysis when combined with the CTSP multivariate correlation statistic, can do much more than deliver products and services to customers. It can also deliver sophisticated multivariate statistical correlation analysis on spreadsheets of data that researcher and engineers encounter on an almost daily basis.

\section{References}

[1] Conley, W.C., Multi stage Monte Carlo optimization applied to systems of integral equations. Proc. of the $15^{\text {th }}$ Int. Conf. on Boundary Element Technology, ed. C.A. Brebbia-WIT Press, Southampton, pp 75-86, 2003.

[2] Anderson, T.W. Multivariate Statistical Analysis, $3^{\text {rd }}$ edition, John Wiley Inc, New York, 2003. 